Check for updates

For numbered affiliations see

end of the article

Correspondence to: M Fallah

m.fallah@dkfz.de

(or @DrFallah1 on Twitter

ORCID 0000-0002-6639-065X)

Additional material is published online only. To view please visit

the journal online.

Cite this as: BMJ 2019;364:1803 http://dx.doi.org/10.1136/bmj.l803

Accepted: 11 February 2019

\section{Familial colorectal cancer risk in half siblings and siblings: nationwide cohort study}

\author{
Yu Tian, ${ }^{1,2^{\star}}$ Elham Kharazmi, ${ }^{1,3^{\star}}$ Kristina Sundquist, ${ }^{3,4,5}$ Jan Sundquist, ${ }^{3,4,5}$ Hermann Brenner, ${ }^{1,6,7}$ \\ Mahdi Fallah ${ }^{1,3}$
}

\section{ABSTRACT}

OBJECTIVE

To explore the risk of colorectal cancer in family members of patients with colorectal cancer, with an emphasis on subtypes of second degree relatives, especially half siblings, which were lacking in the literature.

DESIGN

Ambidirectional cohort study.

SETTING

Nationwide Swedish Family Cancer Data (record linkage).

PARTICIPANTS

All people residing in Sweden and born after 1931, with their biological parents, totalling $>16$ million individuals (follow-up: 1958-2015); of those with clear genealogy, 173796 developed colorectal cancer.

MAIN OUTCOME MEASURES

Lifetime (0-79 years) cumulative risk and standardised incidence ratio of colorectal cancer among first degree relatives and second degree relatives.

RESULTS

The overall lifetime cumulative risk of colorectal cancer in siblings of patients was $7 \%$, which represents a 1.7 -fold (95\% confidence interval 1.6 to $1.7 ; n=2089$ ) increase over the risk in those without any family history of colorectal cancer. A similarly increased lifetime cumulative risk (6\%) was found among half siblings (standardised incidence ratio $1.5,95 \%$ confidence interval 1.3 to $1.8 ; n=140$ ). The risk in people with colorectal cancer in both a parent and a half sibling (standardised incidence ratio 3.6,

\section{WHAT IS ALREADY KNOWN ON THIS TOPIC}

Family history of colorectal cancer in first degree relatives is associated with increased risk of colorectal cancer

Family history of colorectal cancer in second degree relatives has a minor association with the familial risk of colorectal cancer

Although family history is a known risk factor for colorectal cancer, little is known about the risk in different types of relationship in the family history, especially in half siblings

\section{WHAT THIS STUDY ADDS}

Family history of colorectal cancer in half siblings is similarly associated with risk of colorectal cancer to that in siblings

Family history of colorectal cancer in a half sibling (with no affected first degree relative) has a much stronger association with increased risk than in other second degree relatives

The risk of colorectal cancer is substantially increased with family history in one affected second degree relative and an affected first degree relative (or even another second degree relative)
2.4 to $5.0 ; n=32)$ was close to the risk in those with both an affected parent and an affected sibling (2.7, 2.4 to $3.0 ; n=396)$. Family history of colorectal cancer in only one second degree relative other than a half sibling (without any affected first degree relatives), such as a grandparent, uncle, or aunt, showed minor association with the risk of colorectal cancer.

\section{CONCLUSION}

Family history of colorectal cancer in half siblings is similarly associated with colorectal cancer risk to that in siblings. The increase in risk of colorectal cancer among people with one affected second degree relative was negligible, except for half siblings, but the risk was substantially increased for a combination of family history in one affected second degree relative and an affected first degree relative (or even another second degree relative). These evidence based findings provide novel information to help to identify people at high risk with a family history of colorectal cancer that can potentially be used for risk adapted screening.

\section{Introduction}

Colorectal cancer is a major malignant disease of the gastrointestinal tract, which is the third most common cancer and the second leading cause of death from cancer worldwide. ${ }^{1}$ The global burden of colorectal cancer is expected to increase by $60 \%$ to more than 2.2 million new cases and 1.1 million deaths by $2030 .^{2}$ More than 400000 people are diagnosed as having colorectal cancer and more than 200000 die from it every year in Europe, ${ }^{34}$ and colorectal cancer has become the second most common cancer and the second most common cause of death from cancer in Europe. In the US, colorectal cancer is the third most common cancer diagnosed and also the third leading cause of cancer related deaths in both men and women. $^{5}$

Environmental factors play a major role in the causes of colorectal cancer, including cultural, social, and lifestyle practices, resulting in the differences in risk of colorectal cancer between countries and regions depending on the level of economic development, to which an estimated $70-80 \%$ of cases can be attributed. ${ }^{6}$ Germline mutations due to certain cancer related genetic defects, such as Lynch syndrome and familial adenomatous polyposis, account for about $2-5 \%$ of colorectal cancers. The causes of the remaining 20$30 \%$ of inherited colorectal cancers are not completely understood. Colorectal cancer tends to run in families, possibly because of inherited genetic predisposition, shared environmental factors, or the combination of both. ${ }^{7}$ Many epidemiological studies have shown 
increased risk of colorectal cancer among first degree relatives of people with colorectal cancer, ${ }^{89}$ but none for half siblings.

Colorectal cancer screening strategies for people at different risk have been recommended in many guidelines, ${ }^{10-12}$ as colorectal cancer can be detected early by screening and is one of the few cancers that can be prevented with screening by removal of polyps or adenomas. Many studies have shown that methods of screening for colorectal cancer, such as faecal immunochemical test, flexible sigmoidoscopy, and colonoscopy, are effective in reducing its incidence and mortality, and they are more cost effective than no screening. ${ }^{13}$ Increasing screening for colorectal cancer in people at high risk has led to a decline in the incidence of and mortality from colorectal cancer in the US for several decades. ${ }^{5}$ Family history is usually used for risk stratification of colorectal cancer screening in current guidelines. As the increased risk of colorectal cancer in first degree relatives of patients with colorectal cancer is well established, starting screening at younger ages in these people is recommended.

However, some gaps in our knowledge about familial risk of colorectal cancer remain. Few published studies provide information on familial risk of colorectal cancer in second degree relatives. Therefore, to what extent having affected second degree relatives is associated with increased risk of colorectal cancer is still uncertain. A prospective study from Utah assessed the risk of colorectal cancer associated with various combinations of family history, including history of colorectal cancer in second degree relatives. ${ }^{14}$ Another study showed that first degree family history alone is not enough for identification of candidates for high risk screening and suggested future studies on more extended family history (for example, second degree relatives) of colon cancer. ${ }^{15}$ However, none of these studies has provided risk estimates by detailed types of relationship, such as half siblings, grandparent, or uncle/aunt.

The aim of this study was to elucidate whether and to what extent a family history of colorectal cancer in a second degree relative is associated with an increased risk of colorectal cancer and to provide the necessary evidence to help physicians to determine risk of colorectal cancer and make more appropriate recommendations for screening of people with a family history of colorectal cancer. To accomplish this, we analysed the various combinations of family history and further stratified second degree relatives into more detailed relationship types and compared their absolute and relative risks, using the Swedish Family Cancer Data, which is the largest family cancer dataset in the world and is free from the biases of case-control studies.

\section{Methods}

\section{Data linkage}

The datasets used in this study were based on information in the Multi-generation Register, national censuses, the Swedish Cancer Registry, and death notifications (fig 1). ${ }^{16}$ Data on family relationships came from the Multi-generation Register, in which children residing in Sweden and born after 1931 were registered with their biological parents as family units. Thus, the individuals in the datasets can be divided into two generations: offspring (people born after 1931) and their parents. In this nationwide cohort study, the ages of parents were not limited, but offspring were below 84 years of age. This register was linked to the Cancer Registry data (1958-2015) by using the individually unique national registration numbers. Cancer registration in Sweden is based on compulsory reports of cases diagnosed both by clinicians and by pathologists. A four digit diagnostic code is assigned according to the 7th revision of the International Classification of Diseases (ICD-7) and subsequent ICD classifications. No information on the cancer status of individuals was missing in our dataset. We made additional linkages to the national census and death notifications to obtain data on socioeconomic background and deaths. We had full access to the whole pseudonymised population dataset but not to participants. The latest update of the datasets (Swedish family cancer data 2015) includes more than 16 million people, including about two million records of primary invasive cancers. All patients with colorectal cancer in our study had primary invasive colorectal cancer. This record linkage and therefore our exposure and outcomes are updated every two years, which is why our study design is called an ambidirectional cohort study.

\section{Study population}

All people living in Sweden from 1 January 1958 to 31 December 2015 were eligible for the study. Family history was extracted from the linked datasets of the nationwide Cancer Registry and Multi-generation

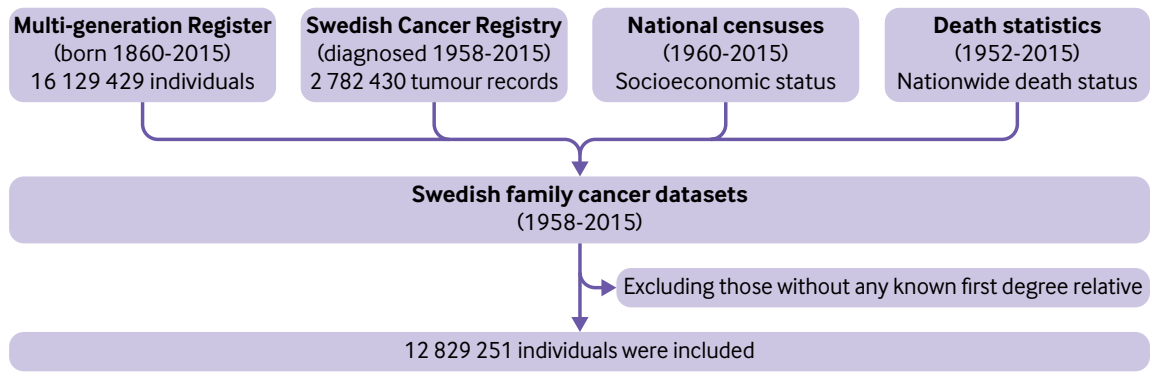

Fig 1 | Flow chart of study population and data sources 
Register (genealogy dataset including unique identification numbers for each person and his/her biological parents). Nobody in the study population had a known second degree relative and unknown first degree relatives, as the former were identified by linkage of the latter. Finally, we included 12829251 people with at least one first degree relative in their genealogy in our analysis.

\section{Statistical analysis}

We calculated standardised incidence ratios to measure the risk of cancers in family members of patients with colorectal cancer as the ratio of observed to expected number of cases. We calculated the expected numbers from strata specific person years in people with a certain family history of colorectal cancer multiplied by strata specific incidence rates in those without a history of colorectal cancer in their first and second degree relatives. For stratification and adjustment, we used five year age group, sex, calendar period (1958-64, 1965-69, 1970-74, ..., 2005-09, and 2010-15), socioeconomic status (blue collar worker, white collar worker, farmer, private, professional, or other/unspecified), and residential area (large cities, small cities in south Sweden, and small cities in north Sweden). We additionally included hospital admission for obesity, alcoholism, and chronic obstructive pulmonary disease (as a proxy for heavy smoking) in our adjustment models. The follow-up started for each family member at birth, immigration date, or 1 January 1958, whichever came latest, and it terminated at the year of diagnosis of colorectal cancer, death, emigration, or the closing date of the study (31 December 2015), whichever came first. We calculated the $95 \%$ confidence intervals of standardised incidence ratios by assuming a Poisson distribution. All family histories reported in our results are exclusive, meaning that, for example, the risk reported for one affected half sibling does not include people with both an affected half sibling and any other affected first or second degree relative.

We calculated the lifetime cumulative risk (assumed to be 0-79 years on the basis of the average life expectancy in Europe in 2015, 78 years ${ }^{17}$ ) by using the following formulas: age specific annual incidence rate=number of cases for each one year age divided by person years for that age; lifetime cumulative rate $=$ sum of all age specific incidence rates by age 79; and lifetime cumulative risk=1-exp(-lifetime cumulative rate), which is expressed as a percentage. We used Poisson approximation to calculate the 95\% confidence intervals of lifetime cumulative risk. We used exact values for person years from individual data (not from conventional aggregated data) in the calculation of cumulative incidences. We used SAS 9.4 for all analyses.

\section{Patient and public involvement}

This was a record linkage study of multiple nationwide datasets. As such, we made no attempts to contact any cohort members for any aspect of this study. Thus, no patients were involved in setting the research question or the outcome measures, nor were they involved in developing plans for recruitment, design, or implementation of the study. No patients were asked to advise on interpretation or writing up of results. There are no plans to directly disseminate the results of the research to study participants. However, dissemination to healthcare professionals, the general public, and relevant patient groups through presentations and other media will be undertaken.

\section{Results}

We included 12829251 people in this study, of which $51 \%(\mathrm{n}=6527022)$ were men. During up to 58 years of follow-up (mean follow-up 33.6 years), 173796 people with available genealogy information (with at least one known first degree relative in our datasets) developed colorectal cancer. In our study, familial colorectal cancer patients (with at least one affected first or second degree relative; $n=16679$ ) constituted approximately $10 \%(n=173796)$ of all patients with colorectal cancer in Sweden.

The familial risk of colorectal cancer, in terms of both standardised incidence ratio and lifetime cumulative risk, increased with the number of affected first degree relatives and second degree relatives (table 1). People with one affected first degree relative (without any affected second degree relatives) had a 1.6-fold (standardised incidence ratio 1.60, 95\% confidence interval 1.57 to 1.63) increased risk of colorectal cancer compared with those with no affected relatives, similar to that in those with two affected second degree relatives without any affected first degree relatives (1.6, 1.4 to 2.0) (table 1). Even the lifetime cumulative risks of these two groups were similar $(6.3 \%$ and $5.7 \%$, respectively). Having a family history in one second degree relative without an affected first degree relative was associated with only a 1.2-fold (95\% confidence interval 1.1 to 1.3 ) increased risk.

Further stratification by type of relationship showed that the lifetime cumulative risk of colorectal cancer in siblings of patients with colorectal cancer was $7 \%$, which represents a 1.7-fold (standardised incidence ratio $1.65,1.58$ to 1.73 ; table 2 ) increase over the $4 \%$ risk in those without any family history of colorectal cancer (table 1). We also found a significantly increased lifetime cumulative risk (6.1\%) among half siblings of colorectal cancer patients (standardised incidence ratio $1.55,1.30$ to 1.83$)$. Further stratification by maternal and paternal lineage of half siblings did not show a statistically significant difference between them (confidence intervals overlapped; table 2).

The risk in people with both a parent and a half sibling with colorectal cancer was greatly increased (standardised incidence ratio 3.6, 2.4 to 5.0), as was the risk in those with both an affected parent and an affected sibling (2.7, 2.4 to 3.0) (table 2). We also found a greatly increased risk of colorectal cancer in people with two affected siblings (standardised incidence ratio 2.2, 1.8 to 2.7), in those with both an affected half sibling and an affected sibling (3.4, 1.4 


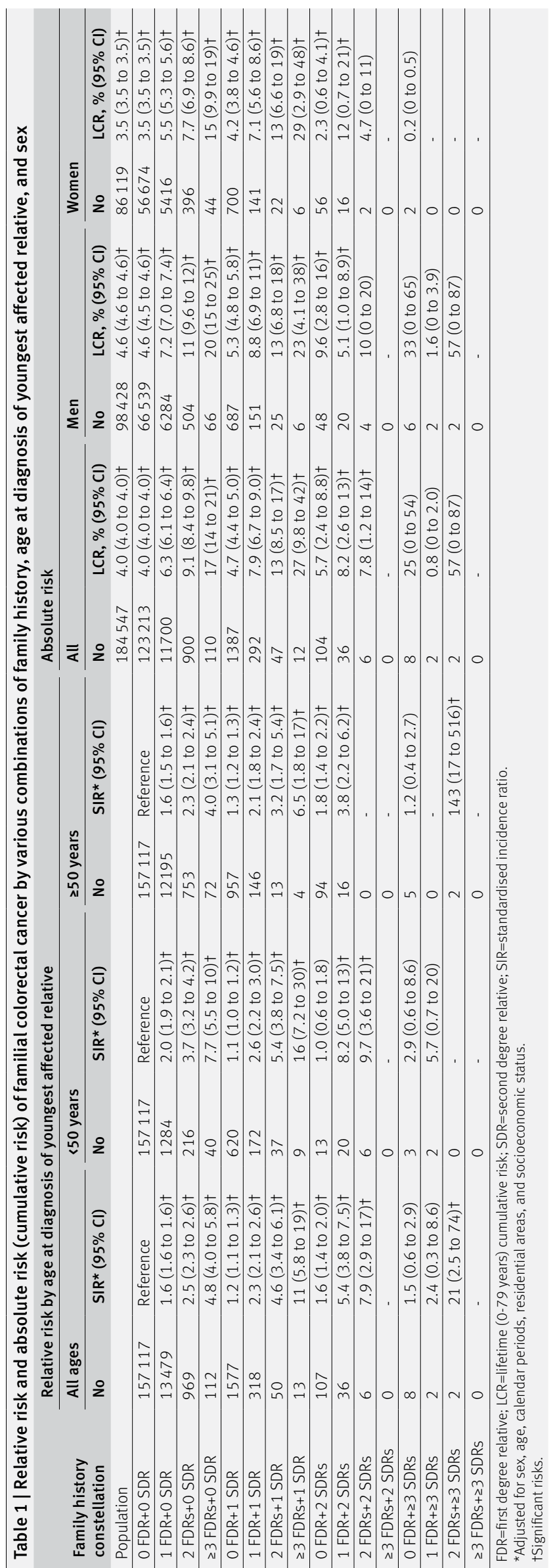

to $6.6 ; \mathrm{n}=8$ ), and even in those with two affected half siblings (3.5, 1.3 to $7.6 ; \mathrm{n}=6)$.

A family history of colorectal cancer in other second degree relatives, such as a grandparent (standardised incidence ratio 1.2, 1.1 to 1.3) or an uncle or aunt (1.2, 1.0 to 1.3 ), without any affected first degree relatives, showed a minor contribution to the familial risk of colorectal cancer (table 2). However, we found higher risks for people with both an affected first degree relative and an affected grandparent (standardised incidence ratio 3.0, 2.4 to 3.7) or both an affected first degree relative and an affected uncle or aunt (2.2, 1.4 to 3.1), which are close to the risk of those with two affected first degree relatives (2.5, 2.3 to 2.6).

We also further analysed the risk in various combinations of family history excluding half siblings, considering the higher risk from affected half siblings than the other second degree relatives mentioned above, and found that the results were only minimally changed in terms of standardised incidence ratio or lifetime cumulative risk, in both sexes (table 3). People with no affected first degree relative but with two affected second degree relatives excluding half siblings (standardised incidence ratio $1.6,1.3$ to 1.9 ) still had a similar risk of colorectal cancer to those with one affected first degree relative (1.60, 1.57 to 1.63).

Further stratification by age at diagnosis of the youngest affected relative showed a higher risk of colorectal cancer when the relative's cancer was diagnosed before age 50 (for example, 2.1-fold for having one affected sibling and 1.9-fold for one affected half sibling) compared with having a late onset of colorectal cancer in relatives (for example, 1.6-fold for one affected sibling and 1.5-fold for one affected half sibling) (table 2). Median age at diagnosis of people with colorectal cancer and only one affected half sibling was 63 years, very close to that of those with one affected sibling (65 years; data not shown). The modification of colorectal cancer risk by age at diagnosis of relatives was less evident for second degree relatives other than half siblings when no first degree relative was affected alongside them (1.1fold for one non-half sibling second degree relative diagnosed before age 50 and 1.2 for one non-half sibling second degree relative diagnosed at older ages; table 3). Further results by age and sex are shown in supplementary tables A, B, and C.

Our stratified analyses by colorectal cancer subsites showed that having a family history of colon cancer was associated with similar colorectal cancer risk between half siblings (1.7-fold) and siblings (1.7fold) (data not shown). Further stratification showed similar trends for family history of cancer in the distal part of the colon (1.7-fold in half siblings and 1.7-fold in siblings) and the proximal part (1.9-fold in half siblings and 1.7-fold in siblings). For family history of rectal cancer, the risk of colorectal cancer was 1.5-fold in siblings (95\% confidence interval 1.4 to 1.7 ) and not statistically significant in a rather small number of half siblings (1.2-fold, 0.9 to 1.7 ), with overlapping confidence intervals. 
Our results did not vary after further adjustment for admission to hospital for obesity, chronic obstructive pulmonary disease, or alcoholism. We also did sensitivity analyses on the stratified calendar period of diagnosis (supplementary table D). The period specific standardised incidence ratios did not vary substantially. Using Amsterdam II criteria, we found 103 likely cases of hereditary non-polyposis colorectal cancer (HNPCC); after we excluded these from our analyses, familial risks of colorectal cancer remained unchanged (data not shown). In another sensitivity analysis, after exclusion of all families who had any HNPCC related cancer other than colorectal, the standardised incidence ratio for one affected sibling changed only slightly from 1.65 (1.58 to 1.73 ) to 1.63 (1.54 to 1.72 ), and the standardised incidence ratio for one affected half sibling remained unchanged (1.5fold, 1.2 to 1.8) (data not shown).

\section{Discussion}

We found that, in addition to first degree relatives of patients with colorectal cancer being at increased risk of this cancer, a family history of colorectal cancer in half siblings is associated with a similar risk of colorectal cancer to that in siblings. In addition, we showed that the risk of colorectal cancer among people with one affected second degree relative was negligible except among half siblings. However, a combination of family history in an affected second degree relative and an affected first degree relative (or even another second degree relative) was associated with a substantially increased risk of colorectal cancer. Although familial risk of colorectal cancer in terms of relative risk (standardised incidence ratio) showed no substantial difference in men and women, the sex difference was evident in terms of absolute (cumulative) risk, which to some extent reflected the global trend of higher incidence of colorectal cancer incidence in men. ${ }^{1}$

\section{Strengths and weaknesses of study}

We were able to further elucidate the familial risk in people who had affected second degree relatives, using the register based nationwide Swedish data, which are the world's largest population based familial cancer datasets, providing novel information to improve counselling for screening for people with a family history of colorectal cancer, with a structure that makes it free from selection and recall bias. We obtained family history from the linked datasets of the Multi-generation Register (genealogy dataset) and nationwide Cancer Registry and not from patients' reports. Collection of family history using record linkage also avoids the bias from under-reporting of self reported family history. ${ }^{18}$ Therefore, our risk estimates should be relatively accurate. Moreover, the familial risk in terms of relative risk (standardised incidence ratio) usually does not substantially change with incidence of cancer; our standardised incidence ratios thus are generalisable to almost all populations. Family size in the reference population (those without family history of colorectal cancer) in general may be 


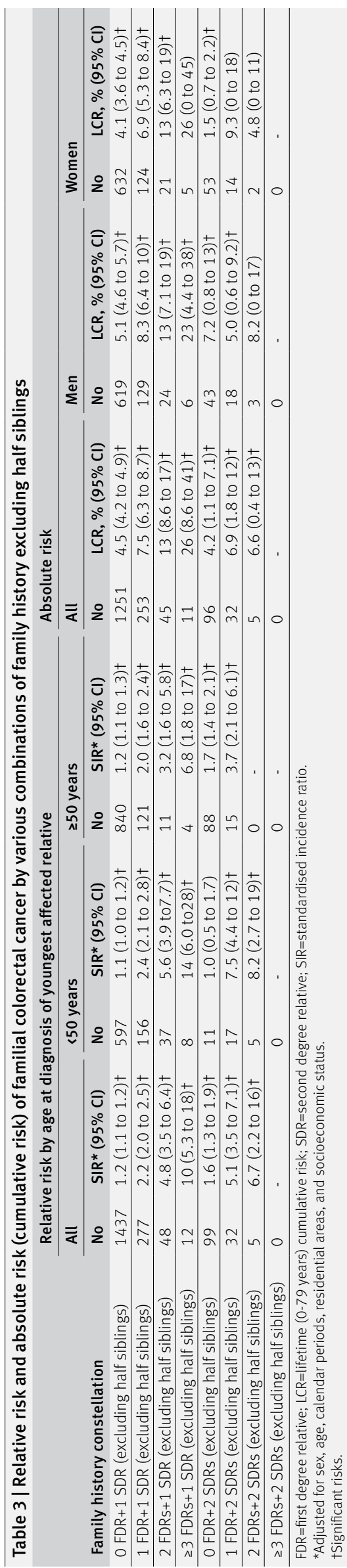

smaller than that in those with a family history of colorectal cancer, and the birth order might influence the risk of some cancers. However, according to previous studies, family size and birth order do not modify the risk of colorectal cancer. ${ }^{19} 20$ As obesity, smoking, and alcoholism are factors associated with risk of colorectal cancer, we made further adjustment for hospital admission related to these factors, which did not change our results substantially. As admission to hospital for these conditions includes only extreme cases, further studies with more complete information on these factors are warranted.

Some information on certain risk factors for colorectal cancer was lacking in our datasets, such as physical activity and dietary habits. We alternatively adjusted our results for residential areas and socioeconomic status, which to some extent contain information on the above mentioned lifestyle related factors. We did not have information on genetic and known hereditary colorectal cancer syndromes, such as HNPCC or familial adenomatous polyposis. However, on the basis of the Amsterdam II criteria for identifying likely HNPCC families, ${ }^{21}$ we tried to identify HNPCC cases in our datasets and found that after exclusion of those likely HNPCC families, or even more strictly exclusion of all the families with any HNPCC related cancer other than colorectal cancer from the analyses, the familial risk of colorectal cancer remained unchanged. As we had no direct data on confirmed cases of HNPCC, a few undetected cases HNPCC may be present among our patients. However, owing to the rarity of such cases, our main results for families are unlikely to be overestimated due to a few cases of HNPCC, especially the standardised incidence ratios for people with few cancer patients in the family, such as only one affected sibling or half sibling.

Colorectal cancer may be detected by screening or by investigation of early symptoms or may remain undetected for some time in people who do not come forward for screening or who ignore early symptoms. Hence, some of the association of cancer with a family history may reflect ascertainment bias. However, this could operate similarly or even less for a history of colorectal cancer in half siblings compared with that in full siblings. Previous Swedish studies on risk of colorectal cancer among adoptees and their adoptive parents (with solely shared environment in childhood and adolescence of the adoptee with the adoptive parents; 1.12 -fold, $95 \%$ confidence interval 0.68 to 1.67) ${ }^{22}$ and spouses (with solely shared adulthood environment; 1.06 -fold, 1.00 to 1.12 ) ${ }^{23}$ suggest that shared environment alone could not explain the risk of colorectal cancer aggregated in family members. Although no nationwide colorectal cancer screening programme exists in Sweden, except for pilot phase screening in Stockholm Gotland region with $8.5 \%$ invitation coverage, ${ }^{24}$ we did a sensitivity analysis by calendar period of diagnosis before and after the colorectal cancer screening era in Sweden, which in general showed no substantial change in familial risk of colorectal cancer (supplementary table D). 
The consistency of our findings across sex, age at diagnosis, calendar period of diagnosis, paternal/ maternal lineage, and colorectal cancer subsites confirms the internal validity of our results. Altogether, the aggregation of risk of colorectal cancer among half siblings seems to be due to a combination of genetic and shared childhood environmental factors.

\section{Comparisons with other studies and implications of findings}

Although several studies have shown an increased risk of colorectal cancer in people with a family history of colorectal cancer in their first degree relatives, ${ }^{89}$ few studies have determined the familial risk of colorectal cancer in second degree relatives, and most of the existing studies on risk of colorectal cancer in second degree relatives failed to present detailed relationship types of the affected second degree relatives and rather used one generalised category of second degree relatives. ${ }^{9}{ }^{14}$ 25-27 In particular, the risk of colorectal cancer in half siblings has not been studied before. We for the first time found that the familial risk of colorectal cancer in half siblings stood out exclusively from that in other types of second degree relatives, and the lifetime cumulative risk and relative risk (standardised incidence ratio) of colorectal cancer in half siblings of patients with colorectal cancer were similar to those of siblings of colorectal cancer patients. In contrast, a family history of colorectal cancer in second degree relatives other than half siblings, such as grandparents or uncles/aunts, did not go along with a relevant increase in risk of colorectal cancer in our study. However, the combination of a family history in one affected second degree relative and an affected first degree relative (or even another affected second degree relative) was associated with a substantially increased risk of colorectal cancer, among which the risk in the combinations involving half siblings were in some instances even higher than combinations with siblings. The reason for the latter could be the random variation due to small sample size (for example, 3.4fold risk in those with both a sibling and a half sibling affected $(n=8)$ or 3.5 -fold risk for two affected half siblings $(n=6)$ versus 2.2-fold risk for two affected siblings $(n=105)$, with overlapping 95\% confidence intervals; table 2). Our results are in line with the findings of a previous study from Utah suggesting that higher numbers of affected first degree relatives show much stronger associations with risk of colorectal cancer than affected second degree relatives alone, but when combined with a family history of colorectal cancer in a first degree relative, family history in a second degree relative can indicate a significantly increased risk of colorectal cancer. ${ }^{14}$

Environmental factors have been known to explain the causation of up to $80 \%$ of colorectal cancers. These include cultural, social, and lifestyle practices, resulting in major differences in risk of colorectal cancer between countries and regions depending on the level of economic development. ${ }^{6}$ On the basis of our finding of similar familial risk between half siblings and siblings, an inference might be drawn that the shared childhood environment and lifestyle may have some association with risk of colorectal cancer. Such a similarity seems to be a very strong argument for environmental dominance of risk even in families at high risk, despite controlling for probable confounders. Shared childhood environment or lifestyle seems to be associated with risk of colorectal cancer not only because of the similar risk estimates between half siblings and siblings but also because of the lower familial risk estimates for other second degree relatives (for example, aunts or uncles alone with no simultaneous shared childhood environment; table 3). A previous study points to the importance of heritable factors in familial colorectal cancer risk in siblings. ${ }^{28} \mathrm{~A}$ recent case-control study has additionally used a genetic risk score combined with family history to predict risk of colorectal cancer. ${ }^{29}$ That study showed that both family history and the so far identified genetic variants carry essentially independent risk information and in combination provide great potential for stratification of colorectal cancer risk. ${ }^{29}$ Large aetiological studies are warranted to identify the possible underlying risk factors that explain our findings.

The recommended starting age of mass screening for colorectal cancer in people at average risk differs in different countries. Of current colorectal cancer screening programmes, Austria has the earliest age at start of screening (40 years), but the UK and Sweden offer screening for people from age 60 (subject to a recent change to age 50 in England and Wales), whereas most other European Union countries recommended age 50 or 55 as the starting age. ${ }^{24}$ The American Cancer Society in its latest updated guideline on colorectal cancer screening recommended age 45 as starting age for people at average risk instead of age 50 as in previous decades, owing to the increasing trend of the incidence of this cancer in younger people in the US. ${ }^{12}$ The reason for the increasing incidence of this cancer in young adults might be a change in social factors-for example, the shift in prevalence of risk factors for colorectal cancer, such as overweight and obesity, towards younger ages. Another reason for the highlighted increase in risk of early onset colorectal cancer could be screening for colorectal cancer in older people, which in some countries such as the US and Germany has controlled the increasing incidence of colorectal cancer but had no effect on the rise in young adults before the age of initial screening.

Colorectal cancer screening programmes in general are effective in reducing the incidence of and mortality from colorectal cancer, and they are more cost effective than no screening. ${ }^{13}$ Several modelling studies have shown that intensive colorectal cancer screening strategies targeting people at increased risk (for example, due to family history) would be cost effective. ${ }^{30}$ Although screening guidelines are recommending screening for all people after a certain age, the next question seems to be "whom to screen early and whom to screen later," which needs 
identification of people at high risk, such as those with a family history who are more prone to early onset colorectal cancer, and also identification of people at low risk who can be screened some years later. In this study, we have shown that people with an affected half sibling have a similar risk to those with an affected sibling, so screening them as early as those with an affected sibling would seem to be fair, whereas those with only one affected grandparent or uncle/aunt may not need to be screened very early.

\section{Conclusions}

This study provides highly valid population based evidence that a family history of colorectal cancer in a half sibling is as predictive as a family history of colorectal cancer in a sibling for the individual colorectal cancer risk estimate, which may point to the importance of the role of shared childhood environment and lifestyle factors in risk of colorectal cancer. On the basis of our results, having a family history of colorectal cancer in a half sibling might be handled similarly to having it in a sibling (or in general a first degree relative). Estimated familial risks from this study can be used in clinical practice regardless of the exact underlying reason for them, and health professionals should be aware of these risks to identify the people at high risk. The understanding of these risks may lead to more practical implications and more effective strategies to reduce the burden of colorectal cancer-for example, by personalised recommendations for early screening and detection of colorectal cancer in people with affected first and/or second degree relatives. We suppose that any further risk stratification beyond what currently exists in the colorectal cancer screening guidelines may lead to more personalised screening and may eventually reduce the morbidity and mortality of colorectal cancer in the family members of patients.

\section{AUTHOR AFFILIATIONS}

${ }^{1}$ Division of Preventive Oncology, German Cancer Research Center (DKFZ) and National Center for Tumor Diseases (NCT), Heidelberg, Germany

${ }^{2}$ Medical Faculty Heidelberg, University of Heidelberg, Heidelberg, Germany

${ }^{3}$ Center for Primary Health Care Research, Lund University, Malmö, Sweden

${ }^{4}$ Department of Family Medicine and Community Health, Department of Population Health Science and Policy, Icahn School of Medicine at Mount Sinai, New York, NY, USA

${ }^{5}$ Center for Community-based Healthcare Research and Education (CoHRE), Department of Functional Pathology, School of Medicine, Shimane University, Japan

${ }^{6}$ Division of Clinical Epidemiology and Aging Research, German Cancer Research Center (DKFZ), Heidelberg, Germany

${ }^{7}$ German Cancer Consortium (DKTK), German Cancer Research Center (DKFZ), Heidelberg, Germany

*Contributed equally

Contributors: YT and EK did the analysis, interpreted the results, and wrote the paper with equal contribution. EK and MF planned and designed the study. All authors approved the final manuscript. KS and IS provided the study material. The corresponding author attests that all listed authors meet authorship criteria and that no others meeting the criteria have been omitted. MF is the guarantor.
Funding: YT has been supported by the China Scholarship Council. The funder had no role in the design, implementation, or interpretation of results of this study.

Competing interests: All authors have completed the ICMJE uniform disclosure form at www.icmje.org/coi disclosure.pdf (available on request from the corresponding author) and declare: no support from any organisation for the submitted work (other than that described above); no financial relationships with any organisations that might have an interest in the submitted work in the previous three years; no other relationships or activities that could appear to have influenced the submitted work.

Ethical approval: The study was approved by the Lund Regional Ethics Committee (2012/795). Consent was not obtained, but the presented secondary data are pseudonymised and there is no risk of identification.

Data sharing: No additional data available.

Transparency: The lead author (the manuscript's guarantor) affirms that the manuscript is an honest, accurate, and transparent account of the study being reported; that no important aspects of the study have been omitted; and that any discrepancies from the study as planned (and, if relevant, registered) have been explained.

This is an Open Access article distributed in accordance with the Creative Commons Attribution Non Commercial (CC BY-NC 4.0) license, which permits others to distribute, remix, adapt, build upon this work non-commercially, and license their derivative works on different terms, provided the original work is properly cited and the use is noncommercial. See: http://creativecommons.org/licenses/by-nc/4.0/

1 Bray F, Ferlay J, Soerjomataram I, Siegel RL, Torre LA, Jemal A. Global cancer statistics 2018: GLOBOCAN estimates of incidence and mortality worldwide for 36 cancers in 185 countries. CA Cancer Clin 2018;68:394-424. doi:10.3322/caac.21492

2 Arnold M, Sierra MS, Laversanne M, Soerjomataram I, Jemal A, Bray F. Global patterns and trends in colorectal cancer incidence and mortality. Gut 2017;66:683-91. doi:10.1136/gutjnl-2015-310912

3 Ferlay J, Autier P, Boniol M, Heanue M, Colombet M, Boyle P. Estimates of the cancer incidence and mortality in Europe in 2006. Ann Oncol 2007;18:581-92. doi:10.1093/annonc/mdl498

4 Ferlay J, Steliarova-Foucher E, Lortet-Tieulent J, et al. Cancer incidence and mortality patterns in Europe: estimates for 40 countries in 2012 Eur J Cancer 2013;49:1374-403. doi:10.1016/j.ejca.2012.12.027

5 American Cancer Society. Cancer Facts \& Figures. 2018. https://www. cancer.org/content/dam/cancer-org/research/cancer-facts-andstatistics/annual-cancer-facts-and-figures/2018/cancer-facts-andfigures-2018.pdf.

6 Boyle P, Langman JS. ABC of colorectal cancer: Epidemiology. BMJ 2000;321:805-8. doi:10.1136/bmj.321.7264.805

7 Jasperson KW, Tuohy TM, Neklason DW, Burt RW. Hereditary and familial colon cancer. Gastroenterology 2010;138:2044-58. doi:10.1053/j.gastro.2010.01.054

8 Frank C, Fallah M, Sundquist J, Hemminki A, Hemminki K. Population Landscape of Familial Cancer. Sci Rep 2015;5:12891. doi:10.1038/ srep12891

9 Butterworth AS, Higgins JP, Pharoah P. Relative and absolute risk of colorectal cancer for individuals with a family history: a meta-analysis. Eur J Cancer 2006;42:216-27. doi:10.1016/j. ejca.2005.09.023

10 Cairns SR, Scholefield JH, Steele RJ, et al, British Society of Gastroenterology, Association of Coloproctology for Great Britain and Ireland. Guidelines for colorectal cancer screening and surveillance in moderate and high risk groups (update from 2002). Gut 2010;59:666-89. doi:10.1136/gut.2009.179804

11 Levin B, Lieberman DA, McFarland B, et al, American Cancer Society Colorectal Cancer Advisory Group, US Multi-Society Task Force, American College of Radiology Colon Cancer Committee. Screening and surveillance for the early detection of colorectal cancer and adenomatous polyps, 2008: a joint guideline from the American Cancer Society, the US Multi-Society Task Force on Colorectal Cancer, and the American College of Radiology. CA Cancer J Clin 2008;58:130-60. doi:10.3322/CA.2007.0018

12 Wolf AMD, Fontham ETH, Church TR, et al. Colorectal cancer screening for average-risk adults: 2018 guideline update from the American Cancer Society. CA Cancer J Clin 2018;68:250-81. doi:10.3322/ caac. 21457

13 Patel SS, Kilgore ML. Cost Effectiveness of Colorectal Cancer Screening Strategies. Cancer Control 2015;22:248-58. doi:10.1177/107327481502200219

14 Taylor DP, Burt RW, Williams MS, Haug PJ, Cannon-Albright LA. Population-based family history-specific risks for colorectal cancer: a constellation approach. Gastroenterology 2010;138:877-85. doi:10.1053/j.gastro.2009.11.044 
15 Solomon BL, Whitman T, Wood ME. Contribution of extended family history in assessment of risk for breast and colon cancer. BMC Fam Pract 2016;17:126. doi:10.1186/s12875-016-0521-0

16 Hemminki K, Ji J, Brandt A, Mousavi SM, Sundquist J. The Swedish Family-Cancer Database 2009: prospects for histology-specific and immigrant studies. Int / Cancer 2010;126:2259-67.

17 World Health Organization. World health statistics 2016: monitoring health for the SDGs, sustainable development goals. 2016. http:// www.who.int/iris/handle/10665/206498.

18 Mitchell RJ, Brewster D, Campbell H, et al. Accuracy of reporting of family history of colorectal cancer. Gut 2004;53:291-5. doi:10.1136/gut.2003.027896

19 Bevier M, Weires M, Thomsen H, Sundquist J, Hemminki K. Influence of family size and birth order on risk of cancer: a population-based study. BMC Cancer 2011;11:163. doi:10.1186/1471-2407-11-163

20 Hemminki K, Mutanen P. Birth order, family size, and the risk of cancer in young and middle-aged adults. Br J Cancer 2001;84:146671. doi:10.1054/bjoc.2001.1811

21 Vasen HF, Watson P Mecklin JP, Lynch HT. New clinical criteria for hereditary nonpolyposis colorectal cancer (HNPCC, Lynch syndrome) proposed by the International Collaborative group on HNPCC. Gastroenterology 1999;116:1453-6. doi:10.1016/S00165085(99)70510-X

22 Sundquist K, Sundquist J, Ji J. Contribution of shared environmental factors to familial aggregation of common cancers: an adoption study in Sweden. Eur J Cancer Prev 2015;24:162-4. doi:10.1097/ (E).0000000000000101

23 Frank C, Fallah M, Ji J, Sundquist J, Hemminki K. The population impact of familial cancer, a major cause of cancer. Int Cancer 2014;134:1899-906. doi:10.1002/ijc.28510
24 Basu P, Ponti A, Anttila A, et al. Status of implementation and organization of cancer screening in The European Union Member States-Summary results from the second European screening report. Int J Cancer 2018;142:44-56. doi:10.1002/ijc.31043

25 Safaee A, Moghimi-Dehkordi B, Pourhoseingholi MA, et al. Risk of colorectal cancer in relatives: a case control study. Indian J Cancer 2010;47:27-30. doi:10.4103/0019-509X.58855

26 Samadder NJ, Curtin K, Tuohy TM, et al. Increased risk of colorectal neoplasia among family members of patients with colorectal cancer: a population-based study in Utah. Gastroenterology 2014;147:814821.e5, quiz e15-6. doi:10.1053/j.gastro.2014.07.006

27 Andrieu N, Launoy G, Guillois R, Ory-Paoletti C, Gignoux M. Estimation of the familial relative risk of cancer by site from a French population based family study on colorectal cancer (CCREF study). Gut 2004;53:1322-8. doi:10.1136/gut.2003.036376

28 Hemminki K, Chen B. Familial risk for colorectal cancers are mainly due to heritable causes. Cancer Epidemiol Biomarkers Prev 2004;13:1253-6.

29 Weig| K, Chang-Claude J, Knebel P, Hsu L, Hoffmeister M, Brenner H. Strongly enhanced colorectal cancer risk stratification by combining family history and genetic risk score. Clin Epidemiol 2018;10:14352. doi:10.2147/CLEP.S145636

30 Leddin D, Lieberman DA, Tse F, et al. Clinical Practice Guideline on Screening for Colorectal Cancer in Individuals With a Family History of Nonhereditary Colorectal Cancer or Adenoma: The Canadian Association of Gastroenterology Banff Consensus. Gastroenterology 2018;155:1325-1347.e3. doi:10.1053/j. gastro.2018.08.017

Web appendix: Supplementary tables 